\title{
Traqueostomía percutánea en una unidad de cuidados intensivos*
}

\author{
Drs. ENRIQUE PÉREZ B. ${ }^{1}$, RICARDO YÁÑEZ M. ${ }^{1}$, ELIZABETH AVILÉS C. ${ }^{1}$, \\ CARLOS ALARCÓN S. ${ }^{2}$, OSVALDO WEISSE A. ${ }^{3}$, MAXIMILIANO CURI T. ${ }^{3}$, \\ JORGE VILLALOBOS Z. ${ }^{4}$, ALEJANDRO PALMA M. ${ }^{1}$, LEOPOLDO VILLARROEL M. ${ }^{1}$ \\ 1 Servicio de Cirugía, Hospital Dr. Sótero del Río. \\ 2 Unidad de Pacientes Críticos, Hospital Dr. Sótero del Río. \\ 3 Interno, Escuela de Medicina, Pontificia Universidad Católica de Chile. \\ 4 Unidad de Broncopulmonar, Hospital Dr. Sótero del Río. \\ Santiago, Chile.
}

\begin{abstract}
Percutaneous tracheostomy in an intensive care unit

Background: Prolonged mechanical ventilation (PMV) is a main factor in a long stay at Intensive Care Units (ICU) in our country. The correct and prompt tracheostomy (TQT) indication has shown significant reductions in ICU stay. The classic open technique has been progressively replaced by the percutaneous TQT. Aims: To describe the experience and the results of percutaneous TQT in a tertiary center ICU. Methods: Prospective study of patients in PMV who underwent a percutaneous tracheostomy in ICU at Hospital Dr. Sótero del Rio, since January 2009 to June 2010. We analyse patient characteristics, waiting time, duration of procedure and complications. Results: The series consist of 130 PMV patients, 101 men, median age 51 (17-22) years old, more frequent diagnoses were neurological disease, cerebrovascular disease and infectious disease. The mean waiting time for the procedure was 1 (0-3) day, the operative time was 11 (3-15) minutes, complications rate was 7.6\%, and the most frequent was local hemorrhage. Discussion: Percutaneous TQT technique is a feasible and safe procedure in ICU at a Tertiary Hospital, with a short waiting time and a low complication rate.
\end{abstract}

Key words: Traqueostomy, percutaneous traqueostomy, Intensive Care Unit, prolonged mechanical ventilation.

\section{Resumen}

Introducción: La ventilación mecánica (VM) prolongada es uno de los factores que prolongan la estadía en la Unidad de Pacientes Críticos, el aumento considerable de la realización de traqueostomías (TQT) ha logrado reducir significativamente la estadía en Unidad de Cuidados Intensivos (UCI). La técnica puede ser abierta (quirúrgica) o percutánea. Objetivos: Describir la experiencia y describir los resultados obtenidos de

\footnotetext{
*Recibido el 5 de agosto de 2010 y aceptado para publicación el 27 de diciembre de 2010.

Correspondencia: Dr. Ricardo Yáñez M.

Fax: 562 3536601. Av. Concha y Toro 3459, $5^{\circ}$ piso, Santiago, Chile. yanezmartinez@gmail.com
} 
la realización de la TQT percutánea en UCI en nuestro centro. Métodos: Estudio prospectivo de todos los pacientes sometidos a VM en quienes se realizó una traqueostomía percutánea en la UCI en el Hospital Dr. Sótero del Río desde enero del año 2009 a junio de 2010. Se analizó características de los pacientes, tiempo de espera, duración del procedimiento y complicaciones. Resultados: La serie fue de 130 pacientes que requerían VM prolongada, 101 hombres, mediana de edad 51 (17-22) años, los diagnósticos más frecuentes fueron enfermedad neurológica, enfermedad cerebrovascular y patología infecciosa. El tiempo de espera para el procedimiento fue de 1 (0-3) días, la duración del procedimiento fue de 11 (3-15) minutos, complicaciones en 7,6\%, siendo hemorragia local la más frecuente. Discusión: La TQT percutánea es una técnica posible de implementar en una UCI, con resultados óptimos, baja tasa de complicaciones y con una resolución oportuna.

Palabras clave: Traqueostomía, traqueostomía percutánea, Unidad de Cuidados Intensivos, ventilación mecánica.

\section{Introducción}

La Unidad de Cuidados Intensivos (UCI) es una de las áreas que genera mayor gasto en salud, entre las estrategias de tratamiento más comúnmente utilizadas en esta unidad se encuentra la ventilación mecánica (VM) y la traqueostomía (TQT), la que es requerida generalmente por pacientes que requieren VM prolongada. Se define VM prolongada como aquella que se extiende más de 21 días por al menos 6 hrs diarias ${ }^{1}$. Durante la última década ha aumentado considerablemente la realización de TQT con lo cual se ha logrado reducir significativamente la estadía en $\mathrm{UCI}^{2}$.

Desde la descripción de la TQT quirúrgica en 1905 por Jackson, luego la realización del procedimiento de manera percutánea por Shelden en 1957 y finalmente la introducción de la técnica percutánea actual por Ciaglia en 1985 hasta la actualidad, ha persistido la evolución del procedimiento en la UCI con la llegada de equipos como el fibrobroncoscopio $^{3-5}$. La TQT percutánea es actualmente una técnica segura, costo efectiva logrando perfilarse como una alternativa factible a la TQT quirúrgica con el beneficio de poder ser realizada en la cama del paciente $e^{6-8}$.

Los objetivos del presente trabajo son describir experiencia del uso de TQT percutánea en UCI y evaluar complicaciones y costos de la realización de esta técnica en nuestro centro.

\section{Pacientes y Método}

Se realizó un estudio prospectivo de todos los pacientes sometidos a ventilación mecánica en quienes se realizó una traqueostomía percutánea en la Unidad de Cuidados Intensivos en el Hospital Dr. Sótero del Río desde enero del año 2009 a junio de 2010. Se registró datos demográficos de cada paciente, diagnóstico principal, duración del procedimiento, tiempo entre la indicación y la realización del procedimiento, complicaciones tempranas y tardías, necesidad de realizar cirugía posterior al procedimiento y mortalidad. Además fue consignado si la intervención fue realizada por un cirujano o por un médico de la UCI.

Los criterios de inclusión y exclusión están detallados en la Tabla 1.

\section{Material}

Se utilizó en todos los pacientes el kit de traqueostomía percutánea por dilatación TRACOE (Figura 1), que consta de:

a) Set de dilatación: bisturí, jeringa, aguja 14 G con catéter de teflón, guía de nitinol, dilatador corto, dilatador curvo, 4 compresas.

b) Cánula de traqueostomía con cuff de baja presión.

\section{Técnica}

Las TP fueron realizadas en la UCI por médicos intensivistas capacitados o por cirujanos. En

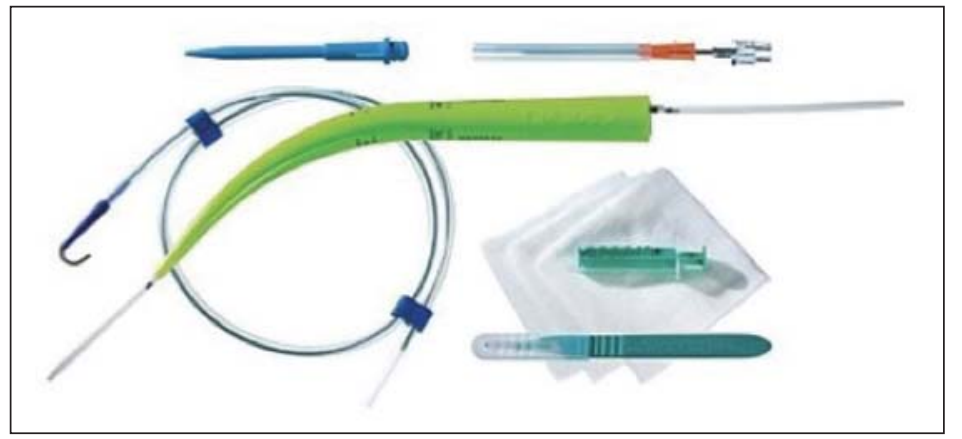

Figura 1. Set de traqueostomía percutánea por dilatación TRACOE con cánula fenestrada. 
los casos en que el paciente fuese más complejo, por ejemplo, pacientes obesos, con edema cervical, difícil palpación de reparos anatómicos cervicales se trató de contar con la ayuda de fibrobroncoscopía, aunque no siempre fue posible contar con el instrumento por motivos administrativos. Durante el procedimiento los pacientes estaban intubados con tubo orotraqueal y ventilación mecánica. Al inicio, a los pacientes se les administró un bolo de sedación fentanilo $100 \mathrm{ug}+$ propofol $1 \mathrm{mg} / \mathrm{kg}$ en caso de tratarse de pacientes hemodinámicamente estables; fentanilo $100 \mathrm{ug}+$ etomidato $0,1 \mathrm{mg} / \mathrm{kg}$ en pacientes inestables.

Posteriormente se aplica clorhexidina en región cervical anterior y con técnica aséptica se crea campo estéril. Según técnica de Ciaglia, se identifica por palpación el espacio ubicado entre el primer y segundo anillo traqueal e infiltra anestésico local. Se realiza incisión cervical anterior transversa de $3 \mathrm{~mm}$ con bisturí y divulsión con pinza Kelly hasta la traquea. Punción traqueal con aguja fina 14 G sobre la que se inserta el catéter de teflón y sobre éste la guía de nitinol. Se retira catéter y se inserta dilatador rígido sobre la guía. Se introduce el dilatador traqueal curvo, previamente lubricado, se retira y se instala cánula de traqueostomía sobre la guía a la vez que se retira el tubo endotraqueal. Se aspira secreciones orofaríngeas previo al retiro del tubo y a través de cánula posterior al retiro del tubo endotraqueal y se conecta a VM. Finalmente, se asegura la cánula con cinta de fijación y 2 puntos de sutura a la piel.

\section{Complicaciones}

Las complicaciones relacionadas a la técnica que fueron evaluadas incluyeron: sangrado de la región operatoria, desaturación/hipoxia, falsa vía, necesidad de traqueostomía abierta, infección de herida operatoria, muerte. No se evaluó neumonías aspirativas.

\section{Análisis estadístico}

Estudio estadístico se realizó con programa SPSS 17.0 y se utilizó t-student para variables continuas y $\chi^{2}$ para variables categóricas con p significativa $<0,05$. Los datos fueron presentados como mediana y rango.

\section{Resultados}

Durante el período analizado se realizó TP a 130 pacientes, 101 hombres. La mediana de edad fue de 51 (17-72) años. Al analizarlos según diagnóstico principal se agrupaban principalmente en traumático (traumatismo encéfalo craneano), cerebrovascular e infeccioso (Tabla 2). El procedimiento fue realizado en 59 casos (45,3\%) por médicos intensivistas y en los restantes 71 (54,6\%) por cirujanos.

Los pacientes intervenidos llevaban en promedio 11 días intubados (rango 6-24 días). El tiempo de espera, es decir, la demora desde que se indicó el procedimiento por el tratante hasta que se realizó la intervención fue de 1 a 3 días.

La duración del procedimiento fue de 11 minutos (rango 3-15) en general; cuando el procedimiento fue realizado por intensivistas la mediana de duración fue 14 minutos (rango 7-15), al realizarlo cirujanos la duración fue de 5,9 minutos (rango 3-15) ( $<<0,005)$, existiendo diferencia significativa al comparar el tiempo entre ambos grupos.

Tabla 1. Criterios de inclusión y exclusión para realización de traqueostomía percutánea en una unidad de pacientes críticos

\begin{tabular}{l}
\hline Criterios de inclusión \\
\hline Ventilación mecánica prolongada \\
Obstrucción de vía aérea alta \\
Alteración reflejos protectores vía aérea \\
Alteración de la deglución \\
\hline Criterios de exclusión \\
\hline Alteración de la coagulación \\
Cirugía cervical previa \\
Tumores cervicales \\
Traumatismo cervical severo \\
Infección local \\
Imposibilidad de palpar puntos anatómicos \\
PEEP $>20$ cm $\mathrm{H}_{2} \mathrm{O}$ \\
\hline
\end{tabular}

Tabla 2. Distribución de los pacientes sometidos a traqueostomía percutánea según diagnóstico

\begin{tabular}{lcc}
\hline Diagnóstico $(\mathbf{n}=\mathbf{1 3 0})$ & $\mathbf{n}$ & $\mathbf{\%}$ \\
\hline Neurológico & 40 & 30,7 \\
Cerebrovascular & 33 & 25,3 \\
Infeccioso & 30 & 23 \\
Intoxicaciones & 9 & 6,9 \\
Cardiovascular & 6 & 4,6 \\
Pancreatitis & 4 & 3,0 \\
Otros & 8 & 6,1 \\
Total & 130 & 100 \\
\hline
\end{tabular}


Fue necesario contar con apoyo fibrobroncoscópico en 26 de los 59 pacientes (44\%) intervenidos por intensivistas y en 23 de los 71 procedimientos $(32,4 \%)$ realizados por cirujanos.

Se registró complicaciones en 10 pacientes $(7,6 \%)$, las cuales fueron hemorragia del sitio operatorio en seis pacientes, hipoxia en un paciente, neumotórax en un paciente y creación de falsa vía en dos pacientes (Tabla 3). Del total de complicaciones, dos ocurrieron cuando el procedimiento fue realizado por cirujanos (falsa vía en un paciente y hemorragia local en otro) y las ocho complicaciones restantes ocurrieron al realizar el procedimiento los médicos intensivistas.

El tratamiento de las complicaciones fue el siguiente: la hemorragia del sitio operatorio fue manejada localmente con ligadura en todos los casos, el caso de hipoxia transitoria fue manejada con aspiración de secreciones bronquiales y del sangrado por fibrobroncoscopía. En los dos pacientes con falsa vía, se registró una desaturación menor de 5 minutos, no presentaron repercusión hemodinámica y se realizó traqueostomía abierta en pabellón con buen resultado. Al paciente con neumotórax se le practicó una pleurostomía sin incidentes.

No se registró complicaciones infecciosas del sitio de la traqueostomía ni del tejido circundante. No hubo asociación estadística entre las complicaciones ocurridas y la edad, ni patología de base del paciente. Hubo una mayor tasa de complicaciones en los primeros 65 pacientes registrándose 11,6\% de complicaciones, luego disminuyó a $5 \%$ en los restantes 65 casos, siendo esta diferencia estadísticamente significativa ( $<<0,005)$.

En ninguno de los pacientes fue necesario volver a realizar intubación orotraqueal, tampoco se registró neumonías aspirativas en este grupo de pacientes. El proceso de weaning posterior a la realización de la TP tuvo una duración promedio de 5 días (rango 3-7 días).

\section{Discusión}

Actualmente en nuestro centro la traqueostomía percutánea es uno de los procedimientos más utilizados en la Unidad de Pacientes Críticos, lo que coincide plenamente con lo expuesto en la literatura, donde se ha visto que la técnica ha sido aceptada ampliamente por los intensivistas, en parte por la baja tasa de complicaciones, donde lo descrito corresponde a un 7-19\%, siendo similar a lo descrito en nuestra experiencia (7,6\% de complicaciones), todas manejadas exitosamente en la cama del paciente, o en pabellón, en el caso de la creación de falsa vía en dos pacientes. Se observó también que existe
Tabla 3. Resumen resultados de la traqueostomía percutánea en una unidad de pacientes críticos

\begin{tabular}{ll}
\hline Traqueostomia percutánea & \\
\hline No Pacientes & 130 \\
Género M/F & $101 / 29$ \\
Edad & 51 (17-72) años \\
Duración procedimiento & 11 min (rango 3-15) \\
Médico UCI & 14 min (rango 7-15) \\
Cirujano & 5,9 min (rango 3-15) \\
Tiempo solicitud-resolución & 1 (0-3) días \\
Complicaciones & 10 pacientes \\
Hemorragia & 6 pacientes \\
Neumotórax & 1 paciente \\
Hipoxia & 1 paciente \\
Falsa vía & 2 pacientes \\
\hline
\end{tabular}

menor número de complicaciones en la medida que se realizan más procedimientos, es decir, existe una curva de aprendizaje, observándose en nuestra experiencia el doble de complicaciones en la primera mitad de los pacientes (11,5\%), lo que disminuyó significativamente en los últimos 65 pacientes. Por lo anterior es importante que cuando la TP es realizada por médicos intensivistas sean ayudados por cirujanos, al menos en los primeros casos, para disminuir el número de complicaciones y optimizar el manejo de estas en caso de que ocurran.

A pesar de haber tenido tres complicaciones importantes, neumotórax y falsas vías, consideramos el procedimiento como seguro para ser realizado en la Unidad de Pacientes Críticos, ya que fueron resueltas adecuadamente y en nuestro centro se cuenta con personal médico capacitado para resolver estas u otras complicaciones relacionadas al procedimiento; por otra parte, las complicaciones importantes descritas ocurrieron en los primeros pacientes, por lo que es de esperar que ocurran con una menor frecuencia. Se ha visto que no existe una diferencia significativa en los resultados obtenidos con la TP al compararlo con la técnica abierta, a corto y largo plazo, excepto porque en algunos estudios se observó mayor infección de herida operatoria en la traqueostomía abierta; sin embargo, a pesar de no haber diferencias significativas, para nuestra realidad la TP ofrece ventajas, tales como evitar la espera por contar con un pabellón para realizar el procedimiento, siendo el tiempo de espera desde la solicitud a la resolución de 1 día en promedio. En general la 
espera desde la solicitud hasta que se realiza la traqueostomía abierta puede variar de entre 1 a 4 días, dependiendo de la disponibilidad de pabellón. Otra ventaja es que el procedimiento puede ser realizado tanto por un intensivista como por un cirujano. Al disminuir los días de espera para la realización de la traqueostomía disminuye también el número de días/cama en UCI.

En base a lo expuesto podemos determinar que la traqueostomía percutánea es una técnica posible de implementar ampliamente en una UCI, con resultados óptimos, baja tasa de complicaciones y a tiempo oportuno.

\section{Referencias}

1. MacIntyre NR, Epstein SK, Carson S, Scheinhorn D, Christopher K, Muldoon S. Management of Patients Requiring Prolonged Mechanical Ventilation. Chest 2005;128:3937-54.

2. Cox CE, Carson SS, Holmes GM, Howard ABS, Carey
TS. Increase in tracheostomy for prolonged mechanical ventilation in North Carolina, 1993-2002. Critical Care Medicine 2004;32:2219-26.

3. Jackson C. Tracheostomy. Laryngoscope 1909;19 28590.

4. Sheldon CH, Pudenz RH, Tichy FY. Percutaneous tracheostomy. JAMA 1957;165:2068-70.

5. Ciaglia P, Frisching R, Syniec C. Elective percutaneous dilatational tracheostomy, a new simple bedside procedure: Preliminary report. Chest 1985;87:715-9.

6. Heikkinen M, Aarnio P, Hannukainen P. Percutaneous dilational tracheostomy or conventional surgical tracheostomy? Critical Care Medicine 2000;28:1399-402.

7. Dulguerov P, Gysin C, Perneger TV, Chevrolet JC. Percutaneous or surgical tracheostomy: a metaanalysis. Crit Care Med. 1999;27:1617-25.

8. Rumbak MJ, Newton M, Truncale T, Schwartz SW, Adams JW, Hazard PB. A prospective, randomized, study comparing early percutaneous dilatational tracheotomy to prolonged translaryngeal intubation (delayed tracheotomy) in critically ill medical patients. Crit Care Med. 2004;32:1689-94. 